\title{
Assessing the performance of data assimilation algorithms which employ linear error feedback
}

Article

Accepted Version

Mallia-Parfitt, N. and Broecker, J. (2016) Assessing the performance of data assimilation algorithms which employ linear error feedback. Chaos, 26 (10). 103109. ISSN 10897682 doi: https://doi.org/10.1063/1.4965029 Available at https://centaur.reading.ac.uk/67574/

It is advisable to refer to the publisher's version if you intend to cite from the work. See Guidance on citing.

To link to this article DOI: http://dx.doi.org/10.1063/1.4965029

Publisher: American Institute of Physics

All outputs in CentAUR are protected by Intellectual Property Rights law, including copyright law. Copyright and IPR is retained by the creators or other copyright holders. Terms and conditions for use of this material are defined in the End User Agreement.

$\underline{\text { www.reading.ac.uk/centaur }}$ 
Central Archive at the University of Reading

Reading's research outputs online 


\section{${ }_{1}$ Assessing the Performance of Data Assimilation Algorithms which employ Linear ${ }_{2}$ Error Feedback \\ $3 \quad$ Noeleene Mallia-Parfitt ${ }^{1}$ and Jochen Bröcker ${ }^{1}$ \\ $4 \quad$ School of Mathematical, Physical and Computational Sciences, 5 University of Reading, Whiteknights, PO BOX 220, Reading, RG6 6AX, \\ $6 \quad$ United Kingdom}

(Dated: 4 October 2016)

Data assimilation means to find an (approximate) trajectory of a dynamical model that (approximately) matches a given set of observations. A direct evaluation of the trajectory against the available observations is likely to yield a too optimistic view of performance, since the observations were already used to find the solution. A possible remedy is presented which simply consists of estimating that optimism, thereby giving a more realistic picture of the 'out of sample' performance. Our approach is inspired by methods from statistical learning employed for model selection and assessment purposes in statistics. Applying similar ideas to data assimilation algorithms yields an operationally viable means of assessment. The approach can be used to improve the performance of models or the data assimilation itself. This is illustrated by optimising the feedback gain for data assimilation employing linear feedback. 
${ }_{8}$ Data assimilation means to find an (approximate) trajectory of a dynamical 9 model that (approximately) matches a given set of observations. A fundamental ${ }_{10}$ problem of data assimilation experiments in atmospheric contexts is that there 11 is no possibility of replication, that is, truly "out of sample" observations from 12 the same underlying flow pattern but with independent observational errors are ${ }_{13}$ typically not available. A direct evaluation against the available observations 14 is likely to yield unrealistic results though, since the observations were already 15 used to find the solution. A possible remedy is presented which simply consists ${ }_{16}$ of estimating that optimism, thereby giving a more realistic picture of the 'out of ${ }_{17}$ sample' performance. The approach is particularly simple when applied to data ${ }_{18}$ assimilation algorithms employing linear error feedback. A realistic performance ${ }_{19}$ assessment is obtained by comparing with the true trajectory. In addition this ${ }_{20}$ method provides a simple and efficient means to determine the optimal feedback ${ }_{21}$ gain operationally since it only requires known quantities to be calculated. The ${ }_{22}$ optimality of this gain is verified numerically. Further, we illustrate theoretical ${ }_{23}$ results which demonstrate that in linear systems with gaussian perturbations, ${ }_{24}$ the feedback thus determined will approach the optimal (Kalman) gain in the ${ }_{25}$ limit of large observational windows (the proof will be given elsewhere).

\section{${ }_{26}$ I. INTRODUCTION}

27 Data Assimilation involves the incorporation of observational data into a numerical model 28 to produce a model state that accurately describes the observed reality. This procedure 29 uses an explicit dynamical model for the time evolution of the observed reality. The results so produced by data assimilation must satisfy two requirements. Firstly they must be close to 31 the observations up to a certain degree of accuracy and secondly they should be consistent 32 with the dynamical model to a certain degree of accuracy. In other words, the trajectory ${ }_{33}$ produced by data assimilation must be close to the observations and it must be close to 34 being an orbit of the model.

35 Once the observations have been used to estimate these trajectories, they should not be 36 used to evaluate the performance of the model (at least not without precaution) as this 
${ }_{37}$ might give unrealistic results. Simply comparing the observations with the output of the 38 data assimilation scheme will provide an overly optimistic picture of performance. Moreover, 39 assessing the performance using this tracking error could easily be cheated. An example is 40 taking the output to be the observations themselves.

${ }_{41}$ As we will see in Section II, a more realistic evaluation of the performance needs to take 42 into account that the output and the observation errors are correlated. To this end, we ${ }_{43}$ investigate the concept of out-of-sample error from statistics and adapt it to the problem of 44 data assimilation. In statistics, estimates of the out-of-sample error are used to measure how 45 well a statistical model, after fitting it to observations, generalises to unseen data ${ }^{1,2}$. Although 46 the concept of the out-of-sample error is a very general one, actual implementations differ ${ }_{47}$ considerably depending on the structure of the estimation problem. Further, a fundamental 48 assumption often made in statistics is that the observations (conditionally on the explanatory ${ }_{49}$ variables) are independent and identically distributed. In the case of linear regression models, 50 a popular statistic for model selection in statistical learning is the $\mathrm{Cp}$ statistic $^{3,4}$. Other ${ }_{51}$ examples are Akaike's Information Criterion (AIC) or the Bayesian Information Criterion ${ }_{52}$ (BIC). These concepts differ in terms of precise interpretation and range of applicability.

${ }_{53}$ The aim of this paper is to provide similar tools in the context of data assimilation. ${ }_{54}$ The underlying problem is essentially the same as in statistics. Suppose a time series of 55 observations has been assimilated into a dynamical model. Then the output should be close 56 to hypothetical observations from the same flow patterns but with independent errors. If ${ }_{57}$ the results are not close to these hypothetical observations, then this can only mean that ${ }_{58}$ the model is in fact not able to explain the dynamics underlying the observations. The 59 out-of-sample error should be a measure of how close the output will be to such hypothetical ${ }_{60}$ observations. Although observations from the same flow pattern but with independent errors ${ }_{61}$ are typically not available in practice, we show that the out-of-sample error can be estimated ${ }_{62}$ using terms that are operationally available. Specifically we show that the out-of-sample ${ }_{63}$ error is the sum of the tracking error and a term which we call the optimism. This optimism ${ }_{64}$ gives us a representation of how the model and observations depend on each other and it ${ }_{65}$ quanties how much the tracking error misestimates the out-of-sample error. The derived ${ }_{66}$ expression is reminiscent of the Cp statistic used in model selection in statistical learning ${ }^{3,4}$. ${ }_{67}$ We show that the optimism takes a very simple form if we assume that the model employs a ${ }_{68}$ linear error feedback. There are many data assimilation algorithms that implement such a 
${ }_{69}$ feedback $^{5}$. More details and references concerning such algorithms can be found in section II. 70 Wahba et al. ${ }^{6}$ apply the ideas of out-of-sample performance to data assimilation for linear 71 systems. In this publication they use generalised cross validation to get an estimate of the 72 true performance. The key equation in this paper is equation (2.11) which is similar to 73 equation (7.46) in Hastie, Tibshirani, and Friedman ${ }^{3}$ with the new aspect being the stochastic 74 approximation to the denominator. The results presented in Wahba et al. ${ }^{6}$ however, apply 75 only in a linear context. As it will be shown, the analysis presented in our paper does not 76 require linear models but merely linear error feedback.

77 We stress that although in terms of the problem we are addressing there is a strong 78 similarity between statistics and data assimilation, our analysis will be different. For instance, 79 although the data assimilation uses linear error feedback, the dependence of the output 80 on the observations as a whole is nonlinear, due to the nonlinearity of the dynamic model. ${ }_{81}$ Further, the observations are not independent. The derivation of the Cp statistic, AIC, ${ }_{82} \mathrm{BIC}$ and many other related concepts used in statistics however assumes either linearity, вз independence or both (see Hastie, Tibshirani, and Friedman ${ }^{3}$, Sec 7.4).

${ }_{84}$ We demonstrate the usefulness of our approach with three numerical examples. In all 85 three cases, we consider a simple data assimilation scheme by means of filtering with a ${ }_{86}$ linear error feedback. A persistent problem in practice is to find a suitable feedback. The 87 feedback acts as a coupling between the true dynamics and the model. If the coupling is too 88 weak the stability of the system cannot be guaranteed while if the coupling is too strong, ${ }_{89}$ results deteriorate because the noise will be overly attenuated. Striking the right balance 90 requires a reliable assessment of the performance which is provided by our estimate of the 91 out-of-sample performance. Note that this is relevant even in the case of linear systems 92 with gaussian perturbations as computing the theoretically optimal Kalman Gain requires 93 knowledge of the dynamical noise which is usually not available in practice. Our experiments 94 demonstrate that the technique can be used in situations where the feedback gain matrix is ${ }_{95}$ completely unspecified and also in situations where it has a pre-determined structure but 96 contains unknown parameters.

${ }_{97}$ In section II we define the tracking error, out-of-sample error and the optimism. These 98 considerations are valid for any data assimilation algorithm in the case of additive observa${ }_{99}$ tional noise. We also consider general data assimilation algorithms which employ linear error 100 feedback and determine an analytical expression for the optimism. Section III contains several 
101 numerical experiments. In Section III A we apply the methodology to a linear system with 102 gaussian perturbations. We minimise an estimate of the out-of-sample error to determine a 103 feedback gain. We then compare this with the asymptotic Kalman Gain which is known to 104 be optimal in this situation. Our experiments suggest that the gain determined numerically 105 agrees with the optimal Kalman Gain in the limit of large observation windows. We discuss a 106 theoretical result which confirms this finding. Next we consider a situation in which the data 107 assimilation algorithm is constrained to have poles in certain locations which determines the 108 gain up to a single parameter. This parameter is determined by minimising an estimate of 109 the out-of-sample error.

110 The remaining experiments consider non linear systems. In Section III B we consider 111 a system in Lur'e form. These systems are special in that, despite being non linear, they 112 permit observers with linear error dynamics. Again a linear feedback is used and we show 113 how an estimate of the out-of-sample error can be used to determine the feedback. The 114 performance of this feedback is assessed numerically by considering the error between the 115 reconstructed and the true orbit. Our results indicate that this strategy of choosing the 116 feedback gives close to optimal performance. Repeating the experiment with the Lorenz '96 117 system in Section III C confirm the results.

\section{${ }_{118}$ II. TRACKING ERROR, OUTPUT ERROR AND OPTIMISM IN DATA ${ }_{119}$ ASSIMILATION}

${ }_{120}$ Data assimilation is the procedure by which trajectories $\left\{z_{n} \in \mathbb{R}^{D}, n=1, \ldots, N\right\}$ (in some 121 state space which we take to be $\mathbb{R}^{D}$ ) are computed with the help of a dynamical model and 122 observations, $\left\{\eta_{n}, n=1, \ldots, N\right\}$. These trajectories should reproduce the observations up to ${ }_{123}$ some degree of accuracy for all $n=1, \ldots, N$. We express this latter part of the procedure 124 formally as: The output $y_{n}=h\left(z_{n}\right)$ is close to the observations $\left\{\eta_{n}, n=1, \ldots, N\right\}$ up to 125 some degree of accuracy, where $h: \mathbb{R}^{D} \rightarrow \mathbb{R}^{d}$ is a function which maps the model's state 126 space into the observation space. This function is usually part of the problem specification. ${ }_{127}$ The exact structure of the model and of $h$ is not important at this stage.

${ }_{128}$ Suppose we have observations $\left\{\eta_{n} \in \mathbb{R}^{d}, n=1, \ldots, N\right\}$ from some real world dynamical 129 phenomenon. We assume $\eta_{n}$ can be written as

$$
\eta_{n}=\zeta_{n}+\sigma r_{n}
$$


130 where $\left\{\zeta_{n}, n=1, \ldots, N\right\}$ are unknown quantities representing the desired signal, and $\sigma \in$ ${ }_{131} \mathbb{R}^{d \times d}$ is the observational error standard deviation. We assume that $\left\{\zeta_{n}, n=1, \ldots, N\right\}$ can 132 be modelled as some stochastic process. The observation errors or noise, $\left\{r_{n}, n=1, \ldots, N\right\}$ 133 are assumed to be independent with mean $\mathbb{E} r_{n}=0$ and variance $\mathbb{E} r_{n} r_{n}^{T}=\mathbb{1}$ and they are 134 independent of $\left\{\zeta_{n}, n=1, \ldots, N\right\}$.

${ }_{135}$ Deviation of the output from the observations can be quantified by means of the tracking 136 error,

$$
E_{T}=\mathbb{E}\left[y_{n}-\eta_{n}\right]^{2} .
$$

${ }_{137}$ The tracking error though is not a very useful performance measure of data assimilation 138 approaches. It is not difficult to design algorithms which achieve zero tracking error by 139 simply using the observations as output, that is any DA algorithm which satisfies $y_{n}=\eta_{n}$, ${ }_{140} n=1, \ldots, N$ achieves optimal performance with respect to $E_{T}$ as a performance measure.

${ }_{141}$ A performance measure which is much harder to hedge is the output error

$$
E_{O}=\mathbb{E}\left[y_{n}-\zeta_{n}\right]^{2} .
$$

${ }_{142}$ A useful relation between $E_{O}$ and $E_{T}$ can be established. Substituting the expression (1) for ${ }_{143}$ the observations into (2) and expanding, we get

$$
E_{T}=\mathbb{E}\left[y_{n}-\eta_{n}\right]^{2}=\mathbb{E}\left[y_{n}-\zeta_{n}\right]^{2}+\operatorname{tr}\left(\sigma^{T} \sigma\right)-2 \operatorname{tr}\left(\sigma \mathbb{E}\left[r_{n} y_{n}^{T}\right]\right)
$$

${ }_{144}$ since $\zeta_{n}$ and $r_{n}$ are independent. The notation 'tr' denotes the trace of the matrix.

145 We re-write this as

$$
E_{O}+\operatorname{tr}\left(\sigma^{T} \sigma\right)=\mathbb{E}\left[y_{n}-\eta_{n}\right]^{2}+2 \operatorname{tr}\left(\sigma \mathbb{E}\left[r_{n} y_{n}^{T}\right]\right) .
$$

${ }_{146}$ The term $2 \sigma \mathbb{E}\left[r_{n} y_{n}^{T}\right]$ is called the optimism. The optimism should be understood as a 147 correlation between $r_{n}$ and $y_{n}$, where $y_{n}$ depends on $\left\{r_{k}, k=1, \ldots, N\right\}$. It is a measure ${ }_{148}$ of how much the tracking error misestimates the output error. We will argue that both 149 the optimism and the tracking error (i.e the first term on the right hand side of (5) can 150 be estimated using operationally available quantities. This will give us a handle on the 151 output error which is, as we have argued, directly related to the true performance of the 152 data assimilation.

${ }_{153}$ The quantity $E_{O}+\sigma^{2}$ can be interpreted as an "Out-of-sample error" as follows: Define 154 hypothetical observations

$$
\eta_{n}^{\prime}=\zeta_{n}+r_{n}^{\prime}, \quad n=1, \ldots, N
$$


155 where $\left\{\zeta_{n}, n=1, \ldots, N\right\}$ is as before, $\left\{r_{n}^{\prime}, n=1, \ldots, N\right\}$ is a process with the same 156 distribution as $\left\{r_{n}, n=1, \ldots, N\right\}$ but independent from it. Then the out-of-sample error is 157 the error between $\left\{y_{n}, n=1 \ldots, N\right\}$ and $\left\{\eta_{n}^{\prime}, n=1, \ldots, N\right\}$, which can be written as

$$
\mathbb{E}\left[y_{n}-\eta_{n}^{\prime}\right]^{2}=E_{O}+\sigma^{2}
$$

${ }_{158}$ The key difference between the tracking error and the out-of-sample error is the absence of 159 correlation between $\left\{y_{n}, n=1 \ldots, N\right\}$ and $\left\{r_{n}^{\prime}, n=1, \ldots, N\right\}$ in the latter, which is precisely 160 the optimism.

${ }_{161}$ Equation (5) shows that the tracking error augmented with further terms, can be a useful 162 measure of performance. Further the tracking error and optimism are relatively easy to 163 estimate. In our experiments we will estimate the tracking error through an empirical average, 164 namely

$$
\hat{E}_{T}=\frac{1}{N} \sum_{k=1}^{N}\left(y_{k}-\eta_{k}\right)^{2} .
$$

165 Estimates of the optimism will be discussed next.

166 We will first calculate a general expression for the optimism for data assimilation schemes 167 which employ a linear error feedback. Most operational data assimilation schemes work in 168 cycles over time. The background field, $\hat{z}_{n}$, is computed at the start of each cycle and usually 169 it is based on information from previous cycles. Since any cycle uses observations available 170 up to that point, the background field at time $n$ only depends on $\eta_{1}, \ldots, \eta_{n-1}$. Nonetheless, 171 the background field $\hat{z}_{n}$ is supposed to be a first guess of the the state of the system at time $172 n$.

173 In this paper we consider data assimilation algorithms which combine the new observation 174 and background through a relationship of the form

$$
z_{n}=\hat{z}_{n}+\mathbf{K}_{n}\left(\eta_{n}-h\left(\hat{z}_{n}\right)\right)
$$

175 where $\mathbf{K}_{n}$ is a $D \times d$ matrix and can depend on $\eta_{1}, \ldots, \eta_{n-1}$ but not on $\eta_{n}$. As before, the 176 mapping $h: \mathbb{R}^{D} \rightarrow \mathbb{R}^{d}$, maps points from model state space to observation space. The 177 modified background, $z_{n}$, is referred to as the analysis.

178 The matrix $\mathbf{K}_{n}$ is the error feedback gain. Equation (9) tells us that the analysis has a 179 linear dependence on the current observation, $\eta_{n}$ and it depends on the previous observations 180 through $\mathbf{K}_{n}$ and $\hat{z}_{n}$. Data assimilation schemes that fall into the presented approach include 
${ }_{181}$ Successive Correction Method (SCM) ${ }^{7,8}$; Optimal Interpolation (OI) ${ }^{9}$; 3D-Var ${ }^{10,11}$; Kalman ${ }_{182}$ Filter variants, ${ }^{12}$ and certain Synchronisation approaches. Synchronisation between dynamical 183 systems has been studied for some time, see for example Pikovsky, Rosenblum, and Kurths ${ }^{13}$; ${ }_{184}$ Huijberts, Nijmeijer, and Pogromsky ${ }^{14}$; Boccaletti et al. ${ }^{15}$. Synchronisation in the setting of 185 data assimilation has also been studied, see Bröcker and Szendro ${ }^{16}$; Szendro, Rodrìguez, and $186 \mathrm{Lopez}^{17}$; Yang, Baker, and $\mathrm{Li}^{18}$. These methods differ only on the approach they take to ${ }_{187}$ calculate the background $\hat{z}_{n}$ and the matrix $\mathbf{K}_{n}{ }^{5}$.

We now consider the optimism as in (5) in the context of DA scheme with linear feedback as in (9). We assume that the function $h\left(x_{n}\right)$ is linear so that $h\left(x_{n}\right)=\mathbf{H} x_{n}$, where $\mathbf{H}$ is a $d \times D$ matrix. Then,

$$
\begin{aligned}
\mathbb{E}\left[r_{n} y_{n}^{T}\right]= & \mathbb{E}\left[r_{n}\left(\mathbf{H} z_{n}\right)^{T}\right]=\mathbb{E}\left[r_{n} z_{n}^{T}\right] \mathbf{H}^{T} \\
= & \mathbb{E}\left[r_{n}\left\{\left(\mathbb{1}-\mathbf{K}_{n} \mathbf{H}\right) \hat{z}_{n}+\mathbf{K}_{n}\left(\zeta_{n}+\sigma r_{n}\right)\right\}^{T}\right] \mathbf{H}^{T} \\
= & \mathbb{E}\left[r_{n}\left(\left(\mathbb{1}-\mathbf{K}_{n} \mathbf{H}\right) \hat{z}_{n}\right)^{T}\right] \mathbf{H}^{T} \\
& +\mathbb{E}\left[r_{n}\left(\mathbf{K}_{n} \zeta_{n}\right)^{T}\right] \mathbf{H}^{T}+\mathbb{E}\left[r_{n}\left(\mathbf{H} \mathbf{K}_{n} \sigma r_{n}\right)^{T}\right] \\
= & \mathbb{E}\left[r_{n} r_{n}^{T} \sigma^{T} \mathbf{K}_{n}^{T}\right] \mathbf{H}^{T} \\
= & \operatorname{tr}\left(\mathbb{E}\left[r_{n} r_{n}^{T}\right] \sigma^{T} \overline{\mathbf{K}}_{n}^{T} \mathbf{H}^{T}\right)
\end{aligned}
$$

188 where $\overline{\mathbf{K}}_{n}=\mathbb{E}\left[\mathbf{K}_{n}\right]$. The first two equalities, (10) and (11), are obtained by substituting the 189 relevant information while (12) is obtained by simply expanding the previous equation. The 190 derivation from (12) to (13) requires some explanation. Notice first that only the third term ${ }_{191}$ of (12) survives. The first term is equal to zero because $\hat{z}_{n}$ and $\mathbf{K}_{n}$ are uncorrelated with $192 r_{n}$. The second term is also equal to zero because $\zeta_{n}$ is independent of $r_{n}$ and because the 193 coupling matrix $\mathbf{K}_{n}$ depends on the observations $\left(\eta_{1} \ldots \eta_{n-1}\right)$ and thus is uncorrelated with ${ }_{194} r_{n}$.

195 Therefore, we are only left with the third term of (12) in (13). Since $\mathbb{E}\left(r_{n} r_{n}^{T}\right)=\mathbb{1},(14)$ 196 implies that

$$
2 \operatorname{tr}\left(\sigma \mathbb{E}\left[r_{n} y_{n}^{T}\right]\right)=2 \operatorname{tr}\left(\sigma \cdot \sigma^{T} \overline{\mathbf{K}}_{n}^{T} \mathbf{H}^{T}\right) .
$$

${ }_{197}$ In the case when $d=1$, which is the case we consider in the numerical experiments later, 198 this reduces to

$$
2 \sigma \mathbb{E}\left[y_{n} r_{n}\right]=2 \mathbf{H} \overline{\mathbf{K}}_{n} \sigma^{2} .
$$


199 We recall that the assumptions necessary to derive this formula are a linear observation 200 operator, $r_{n}$ is independent of $\left\{\eta_{1}, \ldots, \eta_{n-1}\right\}, \mathbb{E} r_{n}=0, \mathbb{E} r_{n} r_{n}^{T}=\mathbb{1}$ and $\mathbf{K}_{n}$ depends only on 201 the observations $\left(\eta_{1}, \ldots, \eta_{n-1}\right)$.

202 In our numerical experiments we approximate the expected value of a random variable by 203 the empirical mean. In particular $E_{T}$ is replaced by its empirical average in (5), resulting in 204 the following estimate for $E_{O}$ for all subsequent numerical experiments (in which $\mathbf{K}_{\mathbf{n}}$ is in 205 fact constant):

$$
\hat{E}_{O}=\hat{E}_{T}+\frac{1}{N} \sum_{n=1}^{N} 2 \sigma^{2} \operatorname{tr}\left(\overline{\mathbf{K}}_{n}^{T} \mathbf{H}^{T}\right)-\sigma^{2} .
$$

206 Let us briefly digress on how the background $\hat{z}_{n}$ and $\mathbf{K}_{n}$ might be calculated in the context 207 of synchronisation, although this is in fact irrelevant for the optimism. Suppose that the 208 reality is given by the non linear dynamical system

$$
\begin{aligned}
x_{n+1} & =\tilde{f}\left(x_{n}\right) \\
\zeta_{n} & =\tilde{h}\left(x_{n}\right) \\
\eta_{n} & =\zeta_{n}+\sigma r_{n}
\end{aligned}
$$

209 where $x_{n} \in \mathbb{R}^{D}$ is referred to as the state and $\zeta_{n} \in \mathbb{R}^{d}$ are the true observations. For this 210 non linear dynamical system we construct a sequential scheme

$$
\begin{aligned}
\hat{z}_{n+1} & =f\left(z_{n}\right) \\
z_{n+1} & =\hat{z}_{n+1}-\mathbf{K}_{n}\left(h\left(\hat{z}_{n+1}\right)-\eta_{n+1}\right) \\
y_{n} & =h\left(z_{n}\right)
\end{aligned}
$$

${ }_{211}$ where $\mathbf{K}_{n}$ is a $D \times d$ coupling matrix which depends on the observations $\eta_{1}, \ldots \eta_{n}$ but ${ }_{212}$ not on $\eta_{n+1}$; and $y_{n}$ is the model output where we hope that $y_{n} \cong \zeta_{n}$. Here $f$ and $h$ are ${ }_{213}$ approximations to the functions $\tilde{f}$ and $\tilde{h}$, respectively. The coupling introduced in this ${ }_{214}$ scheme creates a linear feedback, in the sense that the error between $y_{n}=h\left(\hat{z}_{n}\right)$ and the 215 observations $\eta_{n}$ is fed back into the model.

216 Synchronisation refers to a situation in which, due to coupling, the error $y_{n}-\eta_{n}$ becomes ${ }_{217}$ small asymptotically irrespective of the initial conditions for the model ${ }^{13}$. Often a control ${ }_{218}$ theoretic approach is taken to determine conditions which guarantee the model output, ${ }_{219} y_{n}=h\left(z_{n}\right)$, converging to the observations, $\eta_{n}$ or even $z_{n}$ converging to $x_{n}$ (strictly speaking, 220 the difference converging to zero; note that this can only be expected in case of noise free 221 observations). 
It has been highlighted above that the tracking error is not an ideal measure of performance; 223 however the output error is and moreover, it can be calculated using terms that are readily 224 available. An important question that arises in operational practice is to how to choose 225 the gain matrix $\mathbf{K}$. The numerical experiments detailed below consider different conditions 226 under which to select the appropriate coupling matrix to use in the assimilation. For the 227 first linear experiment we consider arbitrary candidates for the gain matrix, while for the 228 second linear experiment we consider gains that guarantee a certain structure of the system 229 matrix (or more specifically the poles thereof).

\section{III. NUMERICAL EXPERIMENTS}

231 We now demonstrate the usefulness of our approach with three numerical examples. In ${ }_{232}$ Section III A we present the methodology for a linear system with gaussian perturbations. We 233 minimise an estimate of the out-of-sample error to determine a feedback gain and compare 234 this with the asymptotic Kalman Gain which is known to be optimal in this situation.

235 The remaining two experiments concern nonlinear systems. In Section III B we present 236 numerical results for the Hénon Map and in Section III C results are established for the ${ }_{237}$ Lorenz'96 System. Again a linear feedback is used and we show how an estimate of the 238 out-of-sample error can be used to determine the feedback.

239 There is some repetition in the obtained results, however this repetition validates our 240 approach across different experiments. The three systems we consider all use a data assimi241 lation scheme that employs linear error feedback. However the underlying systems in each 242 are different; one is linear, one is in Lur'e form and one is nonlinear. The similarities in the 243 results confirm that our methodology applies to many different dynamical systems.

\section{${ }_{244}$ A. Numerical Experiment 1: Linear Map}

In this first linear example the following experimental setup was used: The reality is given 246 by

$$
x_{n+1}=\underbrace{\left[\begin{array}{cc}
-1 & 10 \\
0 & 0.5
\end{array}\right]}_{\mathbf{A}} x_{n}+\rho q_{n+1}
$$


247 with corresponding observations

$$
\eta_{n}=\mathbf{H} x_{n}+\sigma r_{n}
$$

248 where $\mathbf{H}=\left[\begin{array}{ll}1 & 0\end{array}\right], \zeta_{n}=\mathbf{H} x_{n}$ and $\rho \in \mathbb{R}^{D \times D}$ is the model error standard deviation. We assume ${ }_{249}$ that the model and observations are corrupted by random noise. For these experiments we 250 have $x_{n} \in \mathbb{R}^{2}$ and $\eta_{n} \in \mathbb{R}$. The model errors, $q_{n}$, are assumed to be serially independent ${ }_{251}$ errors with mean $\mathbb{E} q_{n}=0$ and variance $\mathbb{E} q_{n} q_{n}^{T}=\mathbb{1}$.

${ }_{252}$ We set up an observer analogous to our sequential scheme (19),

$$
z_{n+1}=\hat{z}_{n+1}+\mathbf{K}_{n}\left(\eta_{n+1}-\mathbf{H} \hat{z}_{n+1}\right), \quad y_{n}=\mathbf{H} z_{n}
$$

253 where

$$
\hat{z}_{n+1}=\underbrace{\left[\begin{array}{cc}
-1 & 10 \\
0 & 0.5
\end{array}\right]}_{\mathrm{A}} z_{n} .
$$

254 In this case the model is coupled to the observations through a linear coupling term which 255 is dependent on the difference between the actual output and the expected output value ${ }_{256}$ based on the next estimate of the state. For these experiments we will take the coupling ${ }_{257}$ matrix $\mathbf{K}_{n}$ to be constant so from here on we write $\mathbf{K}_{n}=\mathbf{K}$.

${ }_{258}$ The error dynamics in this linear example are given by

$$
\begin{aligned}
e_{n+1} & =x_{n+1}-z_{n+1} \\
& =(\mathbf{A}-\mathbf{K H A}) e_{n}+\mathbf{K} r_{n+1}-(\mathbb{1}-\mathbf{K H}) q_{n+1} .
\end{aligned}
$$

${ }_{259}$ Since the noisy part of the error dynamics (Eq. 24) is stationary, synchronisation can 260 be guaranteed if the eigenvalues of the matrix (A - KHA) all lie within the unit circle. ${ }_{261}$ Synchronisation here means that the error dynamics is asymptotically stationary with finite ${ }_{262}$ covariance. To achieve this, we use a result from control theory, for which we need a few ${ }_{263}$ definitions. Let $\mathbf{H A}=\mathbf{C}$ so that the error dynamics are described by the system matrix ${ }_{264}(\mathbf{A}-\mathbf{K C})$. A pair of matrices $(\mathbf{A}, \mathbf{C})$ is called observable if the observability matrix

$$
\mathcal{O}=\left[\begin{array}{lllll}
\mathbf{C} & \mathbf{C A} & \mathbf{C A}^{2} & \ldots & \mathbf{C A}^{D-1}
\end{array}\right]^{T}
$$

265 has full rank. If this condition holds then the poles of the matrix $(\mathbf{A}-\mathbf{K C})$ can be placed 266 anywhere in the complex plane by proper selection of $\mathbf{K}$. In particular they can be placed 267 within the unit circle ${ }^{19}$. 
In our example, $x_{n} \in \mathbb{R}^{2}$ so our observability matrix is

$$
\mathcal{O}=\left[\begin{array}{ll}
\mathbf{H A} & \mathbf{H A}^{2}
\end{array}\right]^{T}
$$

${ }_{269}$ It is straightforward to check that the linear system we are working with here is observable 270 even though $\mathbf{A}$ is not stable.

271 It is well known in Kalman Filter theory (see for example Anderson and Moore ${ }^{20}$ ) that 272 the optimal gain matrix $\boldsymbol{\kappa}_{n}$ for a linear filter (in the sense of giving least error covariance) is 273 the Kalman Gain which is defined by

$$
\boldsymbol{\kappa}_{n}=\Sigma_{n} \mathbf{H}^{T}\left(\mathbf{H} \Sigma_{n} \mathbf{H}^{T}+\sigma^{2}\right)^{-1}
$$

${ }_{274}$ where $\Sigma_{n}$ is the error covariance matrix defined by $\Sigma_{n}=\mathbb{E}\left[\left(\hat{z}_{n}-x_{n}\right)\left(\hat{z}_{n}-x_{n}\right)^{T}\right]$ and expressed 275 by the following recursive equation,

$$
\Sigma_{n}=\mathbf{A}\left(\Sigma_{n}-\Sigma_{n} \mathbf{H}^{T}\left(\mathbf{H} \Sigma_{n} \mathbf{H}^{T}+\sigma^{2}\right)^{-1} \mathbf{H} \Sigma_{n}\right) \mathbf{A}^{T}+\rho^{2} \cdot \mathbb{1}
$$

${ }_{276}$ Kalman Filter theory states that for $n$ large, the error covariance $\Sigma_{n}$ converges to $\Sigma_{\infty}$ which 277 is the solution to

$$
\Sigma_{\infty}=\mathbf{A}\left[\Sigma_{\infty}-\Sigma_{\infty} \mathbf{H}^{T}\left(\mathbf{H} \Sigma_{\infty} \mathbf{H}^{T}+\sigma^{2}\right)^{-1} \mathbf{H} \Sigma_{\infty}\right] \mathbf{A}^{T}+\rho^{2} \cdot \mathbb{1}
$$

${ }_{278}$ This in turn implies that the Kalman Gain (27) converges to the asymptotic gain which is 279 defined by

$$
\boldsymbol{\kappa}_{\infty}=\Sigma_{\infty} \mathbf{H}^{T}\left(\mathbf{H} \Sigma_{\infty} \mathbf{H}^{T}+\mathbf{R}\right)^{-1}
$$

280 The asymptotic gain, $\boldsymbol{\kappa}_{\infty}$, is obtained by solving the Discrete Algebraic Riccati Equation 281 (DARE) given by (29) and using the solution to calculate (30). Using Maple's inbuilt DARE 282 solver we were able to find the solution to this equation for the experimental setup described 283 above. The Algebraic Riccati Equation is solved using the method described in Arnold III 284 and $\mathrm{Laub}^{21}$.

285 The aim of this experiment is to estimate the optimal gain matrix, $\boldsymbol{\kappa}_{\infty}$ without referring 286 to the DARE, in particular without knowledge of $\rho$. We do this by minimising the empirical ${ }_{287}$ out-of-sample error with respect to $\mathbf{K}$. In other words, our estimate of $\boldsymbol{\kappa}_{\infty}$ is the minimiser of ${ }_{288} \hat{E}_{O}$ for a large (but finite) set of observations (paragraph a. below). This strategy is motivated 289 by our previous discussion about the out-of-sample error being an adequate measure of 290 performance. In fact, in the context of linear systems, we can prove (see Appendix A for 
291 details) that the out-of-sample error is equivalent (in a certain sense) to the asymptotic 292 covariance of $e_{n}$ as a measure of performance. We also stress that estimating the optimism 293 only requires knowledge of $\mathbf{A}, \mathbf{H}, \sigma$ but not $\rho$, the model noise. This is the term that is 294 difficult to determine operationally, so estimating the optimism in an operational situation is 295 possible as all the required terms are readily available. In paragraph b. we discuss a variant 296 of this experiment where the gain matrix is supposed to be optimal under the constraint 297 that the characteristic polynomial has a certain shape.

298 a. Estimating optimal gain matrix The results obtained in this first experiment are 299 shown in Figure 1. The model noise is iid with $\mathbb{E} q_{n}=0, \mathbb{E} q_{n} q_{n}^{T}=1$ and $\rho=0.01$ while 300 for the observational noise, which was also iid with mean zero and variance one, we used ${ }_{301} \sigma=0.1$. We let $n$ vary between zero and $3.5 \times 10^{5}$. For each $n$ the empirical out-of-sample 302 error was minimised and the minimiser was recorded as an estimate of $\boldsymbol{\kappa}_{\infty}$. The experiment 303 was repeated for 100 realisations of the observational noise, $r_{n}$ so that the estimates were 304 different every time. As a measure of accuracy, 90\% confidence intervals were constructed. ${ }_{305}$ We expect that the estimates converge to the asymptotic gain $\boldsymbol{\kappa}_{\infty}$ given by the solution of $306(29,30)$.

307 The results obtained are shown in Figure 1. Figure 1(a) shows a plot in blue squares 308 of the quantity $\left\|\mathbf{K}-\boldsymbol{\kappa}_{\infty}\right\| /\left\|\boldsymbol{\kappa}_{\infty}\right\|$ against $n$. The figure shows that the gain matrix that 309 minimises the out-of-sample error converges exponentially to the asymptotic gain. Moreover, 310 it is illustarted in Figure 1(c) that the eigenvalues of the matrix ( $\mathbf{A}-\mathbf{K H A}$ ) for each gain 311 minimising the out-of-sample error, converge to the eigenvalues of the matrix $\left(\mathbf{A}-\boldsymbol{\kappa}_{\infty} \mathbf{H A}\right)$. 312 Figure $1(\mathrm{c})$ shows the quantity $\left\|\lambda-\lambda_{\infty}\right\| /\left\|\lambda_{\infty}\right\|$ against $n$ in blue diamonds, where $\lambda$ 313 represents the eigenvalues of the matrix (A - KHA). The convergence of the eigenvalues is 314 also exponential. The values of these eigenvalues confirm that the minimising gains stabilise 315 the system since all of then are within the unit circle.

316 The remaining two figures in Figure 1 show a log plot of the same information outlined 317 above. Figure 1(b) represents the convergence of the gain matrices while Figure 1(d) shows 318 the same information for the eigenvalues. Both plots are almost straight lines as expected 319 since the convergence has already been noted to be exponential. The addition to these plots 320 are the $90 \%$ confidence intervals. As previously stated, the experiment was repeated for 100 321 realisations of the observational noise and the plotted confidence intervals represents the 322 uncertainty in the numerical experiment. The lower limit of the error bars was taken at the 


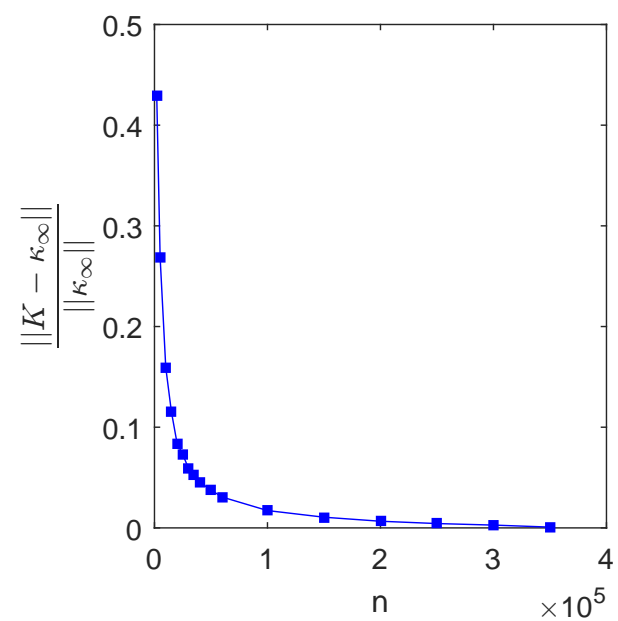

(a)

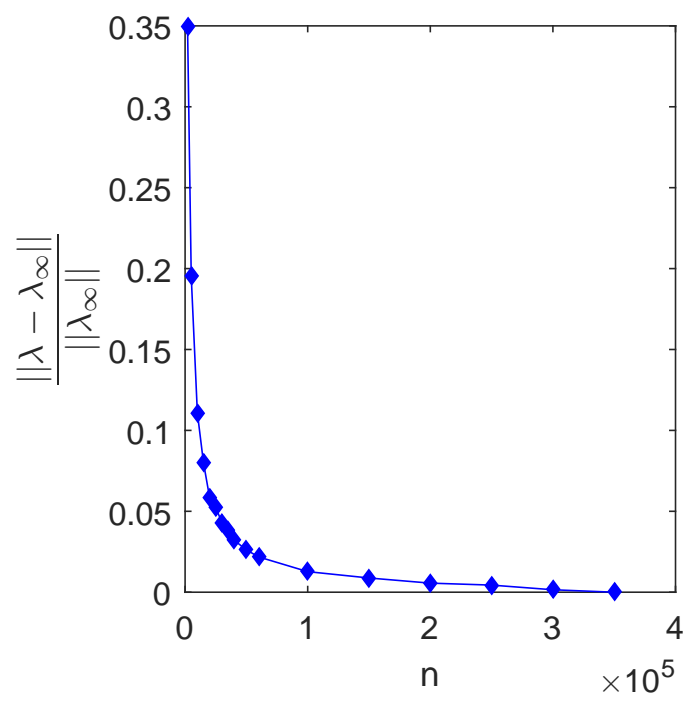

(c)

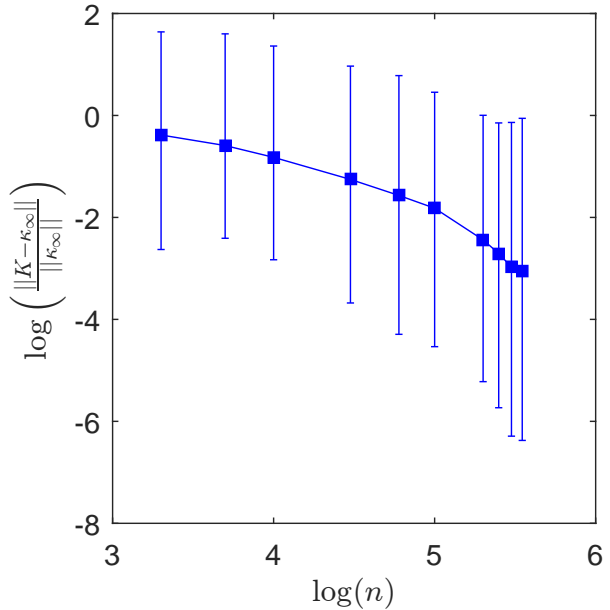

(b)

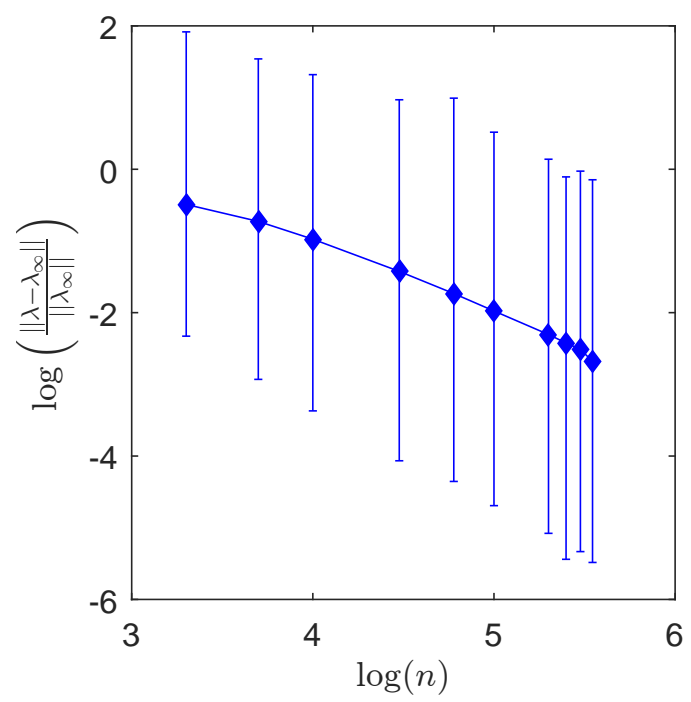

(d)

FIG. 1. Figure 1(a) shows the convergence of the gain minimising the out-of-sample error to the asymptotic gain for increasing $n$. We plot the quantity $\left\|\mathbf{K}-\boldsymbol{\kappa}_{\infty}\right\| /\left\|\boldsymbol{\kappa}_{\infty}\right\|$ against $n$ in blue squares. Figure 1(b) shows a log plot of the same information with $90 \%$ confidence intervals. Figure 1(c) shows the quantity $\left\|\lambda-\lambda_{\infty}\right\| /\left\|\lambda_{\infty}\right\|$ against $n$ in blue diamonds, where $\lambda=\left(\lambda_{1}, \lambda_{2}\right)$ represents the eigenvalues of the matrix $(\mathbf{A}-\mathbf{K H A})$. It is evident that the eigenvalues of the matrix (A - KHA) for each gain minimising the out-of-sample error, converge to the eigenvalues of the matrix (A- $\left.\boldsymbol{\kappa}_{\infty} \mathbf{H A}\right)$, with $n$ increasing. Figure 1(d) shows a log plot of the same information with $90 \%$ confidence intervals. 


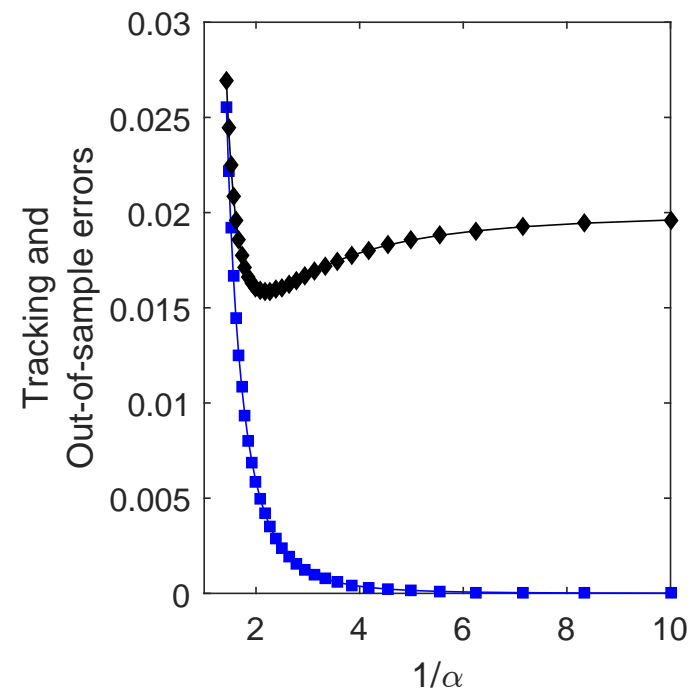

(a)

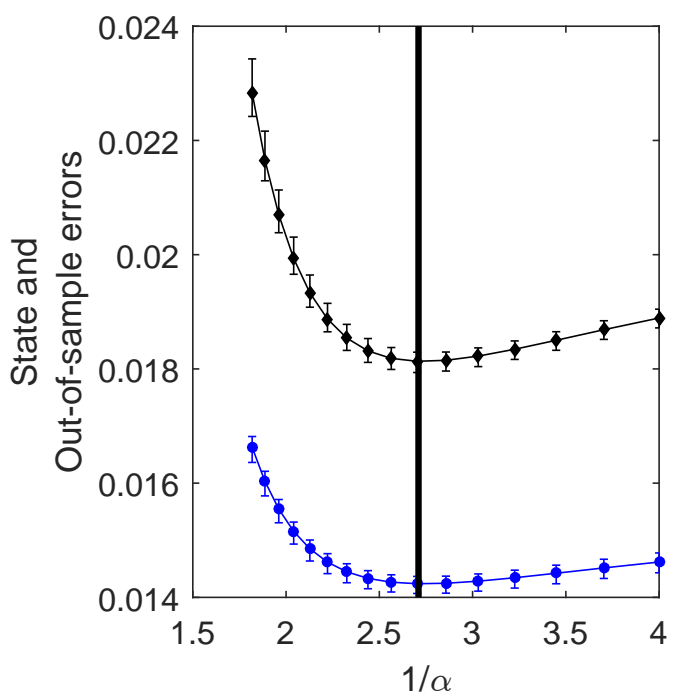

(b)

FIG. 2. Figure 2(a) shows a plot of the tracking error in blue squares and the out-of-sample error in black diamonds. The errors are plotted against the inverse of $\alpha$ for $\sigma=0.1$ and $\rho=0.01$. Figure 2(b) shows a plot of the out-of-sample error in black diamonds for 100 realisations of the noise $r_{n}$ with $\sigma=0.1$ as well as the state error in blue circles. They are displayed for the range of $\alpha$ where the minimum occurs. The error bars in both curves represent $90 \%$ confidence intervals. The black vertical line draws attention to the minimum of the out-of-sample error which coincides with the minimum of the state error.

${ }_{323}$ fifth percentile while the upper limit was taken at the 95 th percentile thus creating the $90 \%$ 324 confidence intervals.

325 b. Gain Matrix with Symmetric Poles In this part of the linear numerical experiment, 326 we want (A - KHA) to have a certain characteristic polynomial. Suppose that the desired 327 characteristic equation is given by

$$
q(\lambda)=(\lambda+\alpha)(\lambda-\alpha)
$$

328 so that $\lambda_{1}=-\lambda_{2}$ and $\left|\lambda_{1}\right|=\left|\lambda_{2}\right|=\alpha$. The appropriate $\mathbf{K}$ for a desired characteristic 329 polynomial, $q(\lambda)$ of the matrix (A - KHA) follows from Ackermann's Formula ${ }^{19}$ which is 330 given by

$$
\mathbf{K}=q(A) \mathcal{O}^{-1}[0 \ldots 1]^{T}
$$

331 where $\mathcal{O}$ is the observability matrix defined in (26). 
The results obtained from our numerical experiment to test the validity of (16) are shown 333 in Figure 2. Figure 2(a) shows a plot of the tracking error in blue squares and the out-of334 sample error in black diamonds. The out-of-sample error calculated via (16) is equivalent to 335 calculating the out-of-sample error explicitly using the output error. We can see that the 336 tracking error tends to zero with decreasing $\alpha$. This is what we expected and is confirmed 337 by using our analytical expression for the optimism.

338 It is clear from Figure 2(a) that while the tracking error tends to zero, the out-of-sample 339 error initially decreases and then increases resulting in a well-defined minimum. This is 340 because as the coupling strength increases, the observations are tracked too closely and thus 341 the output adapts too closely to the observations resulting in an increase of the out-of-sample 342 error. On the other hand when $\alpha$ is large and the coupling strength is weak, the observations 343 are tracked poorly resulting in large tracking and out-of-sample errors. In these experiments ${ }_{344} \alpha$ was varied between 0 and 1 with the assimilation window taken to be $N=10000$.

345 The well defined minimum of the out-of-sample error is also shown in Figure 2(b). ${ }_{346}$ Figure 2(b) shows the out-of-sample error in black diamonds for the range of $\alpha$ where 347 the minimum occurs. The figure shows the out-of-sample error for 100 realisations of the 348 observation noise $r_{n}$ with $\sigma=0.1$ so that the sample estimate is different each time. The 349 error bars in the plot represent $90 \%$ confidence intervals for each value of $\alpha$. The lower 350 limit of the error bars is taken at the fifth percentile, while the upper limit is taken at the 351 95th percentile, hence obtained 90\% confidence intervals as a measure of accuracy. Some 352 further experiments using different values of $\sigma$ where carried out however the results are 353 not included here. The results produced were the same as the ones presented in this paper; 354 the only difference was the size of the error bars produced. A smaller value of $\sigma$ resulted in 355 smaller error bars.

356 To quantify the variation of the parameter $\alpha$ in this experiment, we considered the 357 following calculation. The mean value of the optimal $\alpha$ plus/minus one standard deviation 358 in this case is

$$
\bar{\alpha}^{*} \pm \sqrt{\left(\alpha^{*}-\bar{\alpha}^{*}\right)^{2}}=0.3698 \pm 0.028
$$

359 The second plot in Figure 2(b) illustrates the state error. This estimate of the state error 360 is defined by

$$
\hat{E}_{S}=\frac{1}{N} \sum_{n=1}^{N}\left(z_{n}-x_{n}\right)^{2} .
$$


${ }_{361}$ This is the error that ultimately wants to be analysed and minimised in data assimilation 362 experiments. However, because the model noise $\left(\rho q_{n}\right)$ is difficult to determine, we cannot 363 explicitly analyse the state error which is why we consider errors we can calculate, namely 364 the tracking, output or out-of-sample errors. We can plot the state error $\hat{E}_{S}$ in this example 365 because we have access to it, however in general this is not possible. The vertical line in ${ }_{366}$ Figure 2(b) draws attention to the minimum of the out-of-sample error. It is evident that the 367 state error also has a minimum and the plot suggests that the minima of the out-of-sample 368 and the state error are the same. Again, we ran the experiment for 100 realisations and ${ }_{369}$ plotted the error bars with $90 \%$ confidence intervals.

\section{${ }_{370}$ B. Numerical Experiment 2: Hénon Map}

In this experiment, the reality is given by

$$
x_{n+1}=\underbrace{\left[\begin{array}{ll}
a & b \\
1 & 0
\end{array}\right]}_{\mathbf{A}} x_{n}+c\left[\begin{array}{c}
\left(\mathbf{H} x_{n}\right)^{2} \\
0
\end{array}\right]+d
$$

372 which for the values $a=0, b=0.3, \mathrm{c}=-1.4, d=\left[\begin{array}{ll}1 & 0\end{array}\right]^{T}$ is the chaotic Hénon Map with 373 corresponding observations

$$
\eta_{n}=\mathbf{H} x_{n}+\sigma r_{n}
$$

374 where $\mathbf{H}=\left[\begin{array}{ll}1 & 0\end{array}\right]$, and $\zeta_{n}=\mathbf{H} x_{n}$. The model describing the reality is completely deterministic 375 and we assume that the observations are corrupted by random noise. Notice that we now 376 have a non linear term in the dynamical system. Such systems are said to be in Lur'e form. 377 Once again we consider data assimilation by means of synchronisation so we set up an 378 observer roughly analogous to our sequential scheme (19) with certain differences,

$$
z_{n+1}=\hat{z}_{n+1}+\mathbf{K}_{n}\left(\eta_{n+1}-\mathbf{H} \hat{z}_{n+1}\right), \quad y_{n}=\mathbf{H} z_{n}
$$

379 where

$$
\hat{z}_{n+1}=\underbrace{\left[\begin{array}{ll}
a & b \\
1 & 0
\end{array}\right]}_{\mathrm{A}} z_{n}+c\left[\begin{array}{c}
\eta_{n}^{2} \\
0
\end{array}\right]+d
$$

380 where $a, b, c, d$ are the same as for the reality. In this case as in the first example, the 381 model is coupled to the observations through a linear coupling term which is dependent on 
382 the difference between the actual output and the output value expected based on the next 383 estimate of the state. However there is also a non linear coupling introduced here by the 384 presence of $\eta_{n}^{2}$ in the background term. Note that (16) is still valid nonetheless because $\hat{z}_{n+1}$ 385 is still uncorrelated with $r_{n+1}$. For these experiments we will take the coupling matrix $\mathbf{K}_{n}$ to 386 be constant so from here on in we write $\mathbf{K}_{n}=\mathbf{K}$.

${ }_{387}$ We need to choose the matrix $\mathbf{K}$ appropriately so that we can vary the coupling strength. ${ }_{388}$ For illustration purposes consider the error dynamics for the noise-free situation so that ${ }_{389} \eta_{n}=\mathbf{H} x_{n}$. The error dynamics in this case are given by

$$
\begin{aligned}
e_{n+1} & =x_{n+1}-z_{n+1} \\
& =x_{n+1}-\hat{z}_{n+1}-\mathbf{K H}\left(x_{n+1}-\hat{z}_{n+1}\right) \\
& =(\mathbb{1}-\mathbf{K H})\left(x_{n+1}-\hat{z}_{n+1}\right) \\
& =(\mathbf{A}-\mathbf{K H A})\left(x_{n}-z_{n}\right) \\
& =(\mathbf{A}-\mathbf{K H A}) e_{n} .
\end{aligned}
$$

390 The matrix ( $\mathbf{A}-\mathbf{K H A}$ ) is stable even if $\mathbf{K}=\mathbf{0}$. This means that synchronisation occurs 391 even if there is no linear coupling between the model output and observations because of 392 the non linear coupling introduced in the model (38). The eigenvalues for such a case are ${ }_{393} \lambda_{1,2}= \pm \sqrt{b}$, where $b$ is as in the matrix A. However, it might be that with noise, the 394 out-of-sample error is not optimal for this coupling and can be improved by some additional 395 linear coupling.

396 It is straightforward to check that the system we are working with here is observable 397 provided that $b \neq 0$. The appropriate $\mathbf{K}$ for a desired characteristic polynomial, $q(\lambda)$ of the 398 matrix (A - KHA) again follows from Ackermann's Formula (32). Suppose that the desired 399 characteristic equation is given by

$$
q(\lambda)=(\lambda+\alpha)(\lambda-\alpha)
$$

400 so that $\lambda_{1}=-\lambda_{2}$ and $\left|\lambda_{1}\right|=\left|\lambda_{2}\right|=\alpha$. Then by Ackermann's formula we get

$$
\mathbf{K}=\left[\begin{array}{c}
1-\alpha^{2} / b \\
a \alpha^{2} / b^{2}
\end{array}\right] \Rightarrow \mathbf{H K}=1-\frac{\alpha^{2}}{b}
$$


401 where $a=0$ and $b=0.3$ as in the matrix A. From (41) we see that HK $=1$ if $\alpha=0$. Thus,

$$
y_{n}=\mathbf{H} z_{n}=(\mathbb{1}-\mathbf{H K}) \mathbf{H} \hat{z}_{n}+\mathbf{H K} \eta_{n} \rightarrow \eta_{n},
$$

402 meaning that our data assimilation scheme simply replaces $y_{n}$ with $\eta_{n}$, implying that the ${ }_{403}$ tracking error is zero. In other words, in this example, it is possible to render the eigenvalues 404 of the error dynamics exactly zero and also to obtain zero tracking error. However, the data 405 assimilation is not perfect and the out-of-sample and state errors will not necessarily be 406 small.

407 Therefore, from (16) we know that

$$
\hat{E}_{O}=\hat{E}_{T}-2 \sigma^{2}\left(1-\frac{\alpha^{2}}{b}\right)-\sigma^{2} .
$$

${ }_{408}$ Recall that the aim of this work is to find a way to estimate the out-of-sample error to get a 409 more realistic picture of model performance. We have already determined that when there ${ }_{410}$ is no linear coupling (i.e. $\mathbf{K}=\mathbf{0}$ ) the system is stable and synchronisation occurs. We can ${ }_{411}$ see from (43) that this happens when $\alpha= \pm \sqrt{b}$. There are two further cases to consider. ${ }_{412}$ When $\alpha^{2}>b$ the feedback, due to the linear coupling, is negative. Therefore, in this case 413 we will not be able to improve the out-of-sample error. However as $\alpha$ tends to zero the ${ }_{414}$ optimism will increase and be bounded by $2 \sigma^{2}$. Therefore when $\alpha^{2}<b$ it may be possible to 415 improve the out-of-sample error and determine a coupling matrix $\mathbf{K} \neq \mathbf{0}$, that minimises the 416 out-of-sample error, to be used in the model. We calculate the errors as we did for the linear 417 numerical example in Section III A.

${ }_{418}$ The results obtained from our numerical experiment to test the validity of (16) are shown 419 in Figure 3. Figure 3(a) shows the tracking error in blue squares and the out-of-sample error ${ }_{420}$ in black diamonds. We can see that the tracking error tends to zero with decreasing $\alpha$. This ${ }_{421}$ is what we expected and is confirmed by using our analytical expression for the optimism. ${ }_{422}$ In these experiments $\alpha$ was varied between 0 and 1 with the assimilation window taken to ${ }_{423}$ be $N=10000$.

${ }_{424}$ By analysing the expression for the optimism in this case, we see that there is a point ${ }_{425}$ where the tracking and out-of-sample errors meet. This happens when $\alpha^{2}=b$. To the left of ${ }_{426}$ this, when $\alpha^{2}>b$, the tracking error is greater than the out-of-sample error. To the right, ${ }_{427}$ when $\alpha^{2}<b$, the tracking error is smaller than the out-of-sample error. In fact the tracking ${ }_{428}$ error tends to zero while the out-of-sample error decreases and then starts to increase again 429 resulting in a well defined minimum. 


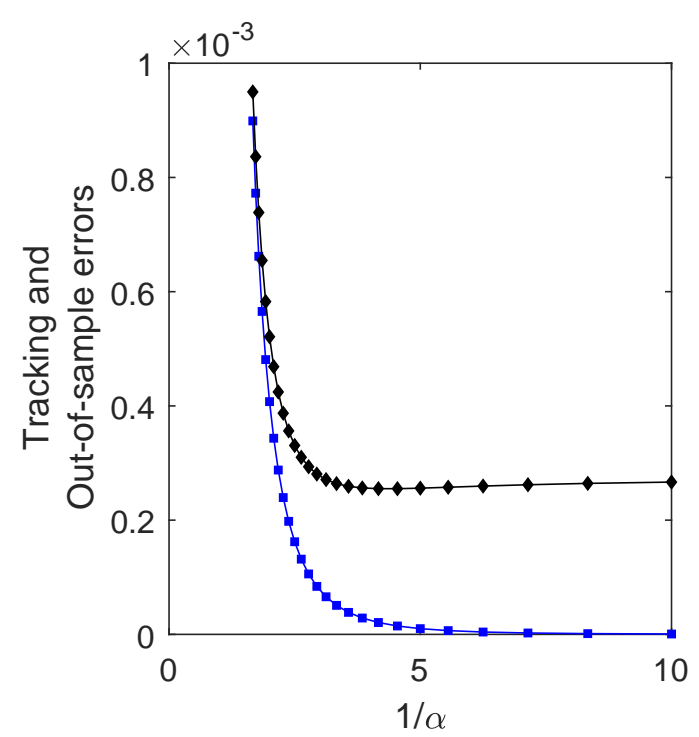

(a)

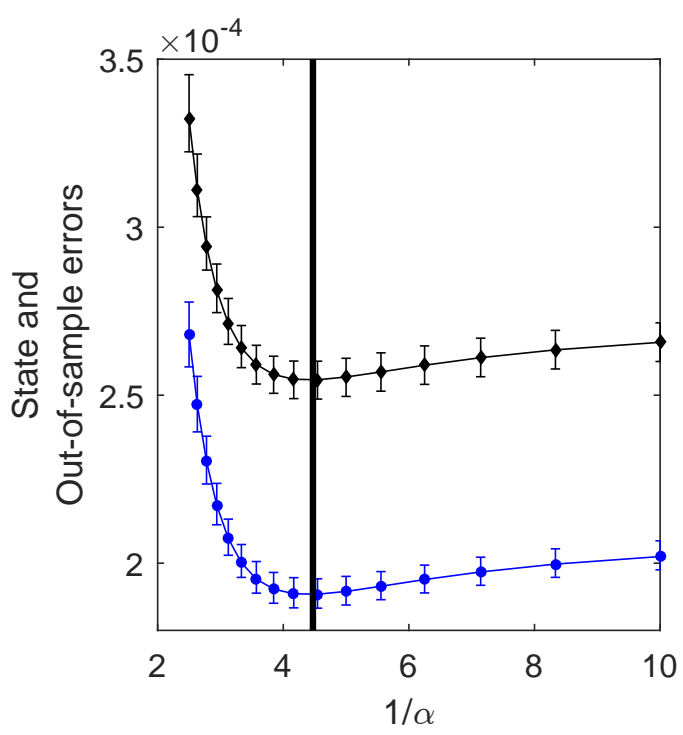

(b)

FIG. 3. Figure 3(a) shows a plot of the tracking error in blue squares and the out-of-sample error in black diamonds. The errors are plotted against the inverse of $\alpha$ for $\sigma=0.01$. Figure 3(b) shows a plot of the out-of-sample error in black diamonds for 100 realisations of the noise $r_{n}$ with $\sigma=0.01$. It is displayed for the range of $\alpha$ where the minimum occurs. The error bars represent $90 \%$ confidence intervals. The state error is show in blue circles also for 100 realisations of the observation noise with $90 \%$ confidence intervals. The vertical line draws attention to the minimum of both curves.

The well defined minimum of the out-of-sample error is shown more clearly in Figure 3(b). ${ }_{431}$ Figure 3(b) shows the out-of-sample error in black diamonds for the range of $\alpha$ where the 432 minimum occurs. The figure shows the out-of-sample error for 100 realisations of the noise ${ }_{433} r_{n}$ for $\sigma=0.01$. The error bars represent $90 \%$ confidence intervals for each $\alpha$. Once again we ${ }_{434}$ would like to quantify the variation of the parameter $\alpha$. The mean value of the optimal $\alpha$ $435 \mathrm{plus} / \mathrm{minus}$ one standard deviation in this case is

$$
\bar{\alpha}^{*} \pm \sqrt{\left(\alpha^{*}-\bar{\alpha}^{*}\right)^{2}}=0.2238 \pm 0.0079
$$

Figure 3(b) also shows a plot of the state error in blue circles for 100 realisations. The ${ }_{437}$ black, vertical line draws attention to the minimum of both curves. We can see that the 438 minimising gain is the same for both errors. When running data assimilation schemes, the 439 state error is the error we are interested in minimising, however we only have access to the 440 error in observation space. Even though this is the case, we have shown numerically that the 
${ }_{441}$ minimising gain is the same for both errors, even in this non linear situation.

${ }_{442}$ As with the linear numerical experiment presented in Section III A, further experiments ${ }_{443}$ using different values of $\sigma$ where carried out. The results produced were the same as the 444 ones presented here; the only difference was the size of the error bars produced. A smaller ${ }_{445}$ value of $\sigma$ resulted in smaller error bars much like it did for the linear numerical example. ${ }_{446}$ What is particularly of interest here is that even though the dynamical system included ${ }_{447}$ a non linear term, the methodology still applies, provided that the matrix ( $\left.\mathbf{A}-\mathbf{K H A}\right)$ is ${ }_{448}$ stable. As an aside, the experiment suggests that the eigenvalues of the linear part of the ${ }_{449}$ error dynamics have to be $<1-\epsilon$ with some small but non-zero $\epsilon$ in order to stabilise the 450 error dynamics.

\section{${ }_{451}$ C. Numerical Experiment 3: Lorenz '96}

452 For this third numerical experiment, the reality is given by the Lorenz'96 model which is ${ }_{453}$ governed by the following equations

$$
\dot{x}_{i}=-x_{i-1}\left(x_{i-2}-x_{i+1}\right)-x_{i}+F
$$

454 and exhibits chaotic behaviour for $F=8$. By integrating the above differential equation with 455 a time step $\delta=1.5 \times 10^{-2}$, we obtain a discrete model for our reality which we denote by

$$
x_{n+1}=\Phi\left(x_{n}\right) .
$$

${ }_{456}$ We take corresponding observations of the form

$$
\eta_{n}=\mathbf{H} x_{n}+\sigma r_{n}
$$

${ }_{457}$ where $\mathbf{H}$ is the observation operator and $r_{n}$ is iid noise. We shall take the state dimension to ${ }_{458}$ be $D=12$, the observation space to be $d=4$ and we define the observation operator so that 459 we observe every third element of the state; that is $\left(x_{1}, x_{4}, x_{7}, x_{10}\right)$. The system we construct 460 here is fully non-linear with linear observations.

${ }_{461}$ The assimilating model will use the Lorenz'96 model coupled to the observations through ${ }_{462}$ a simple linear coupling term, as done in the the previous numerical experiments. We set 463 the coupling matrix $\mathbf{K}$, to be defined by

$$
\mathbf{K}=\kappa \mathbf{H}^{T}
$$




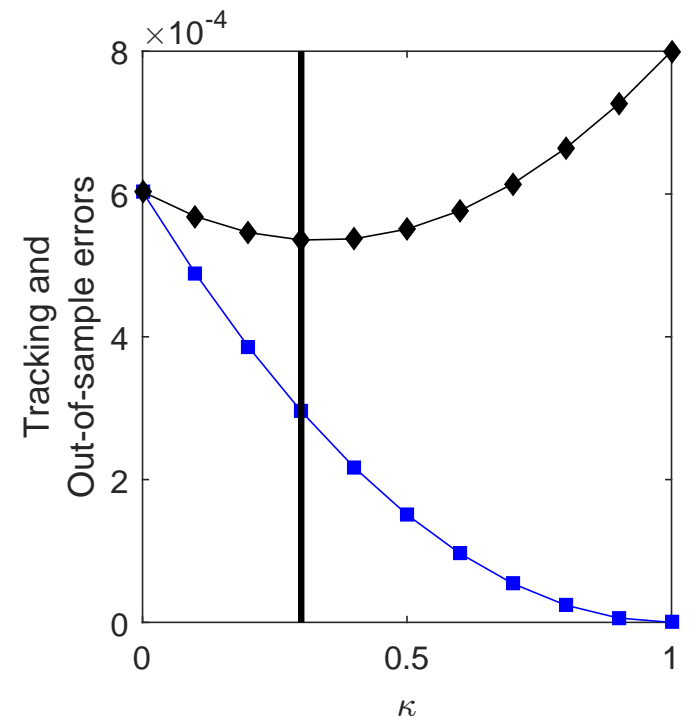

(a)

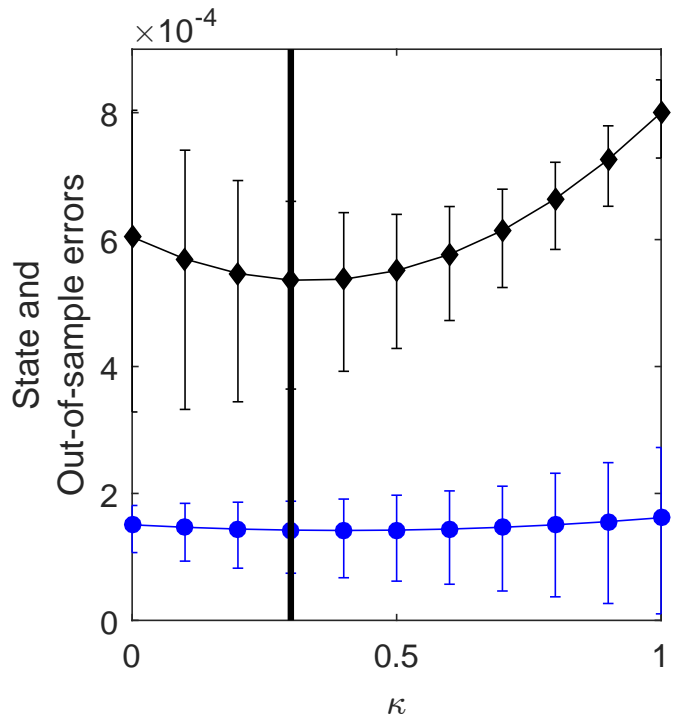

(b)

FIG. 4. Figure 4(a) presents the out-of-sample error (black diamonds) and the tracking error (blue squares). Figure 4(b) illustrates the out-of-sample error (black diamonds) and the state error (blue circles) with the error bars representing $90 \%$ confidence intervals. The black vertical line draws attention to the minimum of the out-of-sample error.

${ }_{464}$ where $\kappa$ is a coupling parameter taken to be between 0 and 1 . With this information, the 465 assimilating model is defined by the following equations

$$
\hat{z}_{n+1}=\Phi\left(z_{n}\right) ; \quad z_{n+1}=\hat{z}_{n+1}+\kappa \mathbf{H}^{T}\left(\eta_{n+1}-\mathbf{H} \hat{z}_{n+1}\right)
$$

${ }_{466}$ Once again we will vary the coupling strength in the observer by adjusting the coupling ${ }_{467}$ parameter $\kappa$. If the coupling is too strong, the observations will be tracked too rigorously and 468 so the observational noise will not be filtered out. If the coupling is too weak the observations 469 are tracked poorly; so once again we expect the out-of-sample error to take a minimum at 470 some non-trivial value of $\kappa$.

471 As always we are interested in the behaviour of the state error and, ultimately, this is the ${ }_{472}$ error we want to be minimal. We saw in Section III B that the minimiser for the out-of-sample 473 error was the same as for the state error. We investigate this here too.

${ }_{474}$ The results obtained are shown in Figure 4. Once again the observational noise is iid with ${ }_{475} \mathbb{E} r_{n}=0, \mathbb{E} r_{n} r_{n}^{T}=1$ and $\sigma=0.01$. Since the gain is given by equation (48), the optimism 476 reduces to $8 \sigma^{2} \kappa$. To see this note that the observation operator, $\mathbf{H}$, was defined so that 477 every third element of the state was observed. It follows then that $\mathbf{H H}^{T}=\mathbb{1}$, the identity 
478 matrix. Since we are observing four states, the trace of $\mathbf{H H}^{T}$ is equal to four. Thus, since 479 the optimism is defined by $2 \sigma^{2} \operatorname{tr}(\mathbf{H K})$ and $\mathbf{K}$ is given by equation (48), it follows that the 480 optimism reduces to $8 \sigma^{2} \kappa$.

${ }_{481}$ To calculate the the errors, a transient time was ignored to give the system time to ${ }_{482}$ synchronise. In Figure 4(a) the out-of-sample error (black diamonds) is presented together ${ }_{483}$ with the tracking error (blue squares). The black vertical line draws the eye to the minimum 484 of the out-of-sample error. As in the previous experiments, the tracking error reduces to zero 485 while the out-of-sample error increases eventually with increasing coupling strength.

${ }_{486}$ Figure 4(b) presents the out-of-sample error (black diamonds) and the state error (blue ${ }_{487}$ circles). The figure shows the errors for 100 realisations of the observational noise, $r_{n}$. The ${ }_{488}$ error bars represent $90 \%$ confidence intervals for each value of $\kappa$ with the lower limit of the ${ }_{489}$ error bars taken at the fifth percentile and the upper limit taken at the 95th. The mean 490 value of the optimal $\kappa$ plus/minus one standard deviation in this case is

$$
\bar{\kappa}^{*} \pm \sqrt{\left(\kappa^{*}-\bar{\kappa}^{*}\right)^{2}}=0.3050 \pm 0.1184 .
$$

491 The black line draws attention to the minimum of the out-of-sample error and we once 492 again see that the minima of the state and out-of-sample errors coincide. It is evident here ${ }_{493}$ that these results support the results determined previously in the numerical experiments. ${ }_{494}$ Further experiments using different values of $\sigma$ where also carried out for this non linear ${ }_{495}$ system. The results produced were the same as the ones presented here; the only difference 496 was the size of the error bars produced. Again, as with the results in the previous two ${ }_{497}$ experiments, a smaller value of $\sigma$ resulted in smaller error bars.

${ }_{498}$ The flatness of the curves and the uncertainty shown in the figures are rather deceptive in ${ }_{499}$ the plots presented in this paper. By looking at these figures, one might expect that the 500 errors in the estimate of $\boldsymbol{\kappa}^{*}$ are in fact quite large. However this is not the case as it is the ${ }_{501}$ correlation between the errors in the plots that matters.

\section{${ }_{502}$ IV. CONCLUSIONS}

${ }_{503}$ A fundamental problem of data assimilation experiments in atmospheric contexts is that 504 there is no possibility of replication, that is, truly "out of sample" observations from the ${ }_{505}$ same underlying flow pattern but with independent observational errors are typically not 
506 available. A direct evaluation of assimilated trajectories against the available observations is 507 likely to yield optimistic results though, since the observations were already used to find the 508 solution.

509 A possible remedy was presented which simply consists of estimating that optimism, 510 thereby giving a more realistic picture of the 'out of sample' performance. The optimism 511 represents the correlation between the observations and the output of the data assimilation 512 scheme. This estimate depends on the observational noise, the observation operator and the 513 feedback gain matrix but not on the underlying dynamics or dynamical noise parameters. ${ }_{514}$ The model noise is the term that is difficult to determine operationally, so estimating the 515 optimism in an operational situation is possible as all the required terms are readily available. 516 In this paper, this approach was applied to data assimilation algorithms employing linear error 517 feedback. Several numerical experiments concerning both linear and non-linear systems give 518 evidence to the success of this method as it provides more realistic assessment of performance. ${ }_{519}$ This was demonstrated by comparing the out-of-sample performance with the true state 520 error of the algorithm which was available in these numerical simulations.

${ }_{521}$ The approach outlined above also provides a simple and efficient means to determine the 522 optimal feedback gain by optimising the out-of-sample error with respect to the gain matrix. ${ }_{523}$ Further, theoretical results demonstrate that in linear systems with gaussian perturbations, 524 the feedback thus determined will approach the optimal (Kalman) gain in the limit of large 525 observational windows. The numerical experiments presented in this paper support this 526 result for linear systems.

${ }_{527}$ We cannot deduce the same thing for the non-linear systems since firstly, we do not have 528 a candidate for the asymptotic error or gain since the Kalman Filter equations do not hold 529 in these cases. Secondly, even if the existence of an optimal asymptotic gain could be proved, 530 the sequence of minimisers might not converge to it.

${ }_{531}$ As an outlook for future work, it seems that the presence of dynamical noise in the 532 underlying system is important when considering the convergence of the optimal gain matrix 533 for non-linear systems. (Even in the linear case, the presence of nondegenerate dynamical ${ }_{534}$ noise is essential for the proof to work). If there is no model noise present, then we cannot 535 expect the gain matrix to converge in a meaningful way as the optimal asymptotic gain may 536 not be well defined. For example it is possible that the dynamics of both the underlying 537 system and model enter a region of stability, resulting in a reduction of the error. In this 
${ }_{538}$ case it would make sense to reduce or completely eliminate the feedback gain matrix. This 539 would need the gain matrix to be adaptive in some way; a concept not considered here.

\section{${ }_{540}$ ACKNOWLEDGMENTS}

${ }_{541}$ This paper was prepared with the support of the Engineering and Physical Sciences ${ }_{542}$ Research Council for Jochen Bröcker under first grant agreement EP/L012669/1. The ${ }_{543}$ authors wish to thank Peter Jan van Leeuwen for helpful discussions and constructive 544 suggestions which motivated some of the work in this paper.

\section{${ }_{545}$ Appendix A}

${ }_{546}$ In this appendix, we want to clarify the relationship between the output error

$$
E_{O, n}=\mathbb{E}\left[\left(\mathbf{H}\left(x_{n}-z_{n}\right)\right)^{2}\right]
$$

${ }_{547}$ (which we give an index $n$ here as it depends on $n$ ) and the error covariance matrix

$$
\Gamma_{n}=\mathbb{E}\left[\left(x_{n}-z_{n}\right)\left(x_{n}-z_{n}\right)^{T}\right]
$$

${ }_{548}$ in the context of linear systems (Section III A). Re-writing the output error we obtain

$$
\begin{aligned}
E_{O, n} & =\mathbb{E}\left\{\left(\mathbf{H}\left(x_{n}-z_{n}\right)\right)^{T}\left(\mathbf{H}\left(x_{n}-z_{n}\right)\right)\right\} \\
& =\mathbb{E} \operatorname{tr}\left\{\left(\mathbf{H}\left(x_{n}-z_{n}\right)\right)^{T} \mathbf{H}\left(x_{n}-z_{n}\right)\right\} \\
& =\mathbb{E} \operatorname{tr}\left\{\mathbf{H}\left(x_{n}-z_{n}\right)\left(x_{n}-z_{n}\right)^{T} \mathbf{H}^{T}\right\} \\
& =\operatorname{tr}\left\{\mathbf{H} \Gamma_{n} \mathbf{H}^{T}\right\}
\end{aligned}
$$

${ }_{549}$ and if we assume real values observations (i.e $d=1$ ), we get $E_{O, n}=\mathbf{H} \Gamma_{n} \mathbf{H}^{T}$. This does not 550 mean that $E_{O, n}$ carries the same information as $\Gamma_{n}$ since $\mathbf{H}$ is not invertible.

${ }_{551}$ To investigate this further, introduce the mappings $F: \mathbb{R}^{D} \times \mathbb{R}^{D \times D} \rightarrow \mathbb{R}^{D \times D},(\mathbf{K}, \mathbf{M}) \rightarrow$ ${ }_{552}(\mathbf{A}-\mathbf{K H A}) \mathbf{M}(\mathbf{A}-\mathbf{K H A})^{T}$ and $G: \mathbb{R}^{D} \rightarrow \mathbb{R}^{D \times D} ; \mathbf{K} \rightarrow \sigma^{2} \mathbf{K K}^{T}+\rho^{2}(\mathbb{1}-\mathbf{K H})(\mathbb{1}-\mathbf{K H})^{T}$ 553 and $\Phi(\mathbf{K}, \mathbf{M})=F(\mathbf{K}, \mathbf{M})+G(\mathbf{K})$. Note that $F$ is linear in $\mathbf{M}$, and we will write $F(\mathbf{K}) \cdot \mathbf{M}$ 554 to emphasize this. It follows from linear filter theory that

$$
\begin{aligned}
\Gamma_{n+1} & =(\mathbf{A}-\mathbf{K H A}) \Gamma_{n}(\mathbf{A}-\mathbf{K H A})^{T}+\sigma^{2} \mathbf{K K}^{T}+\rho^{2}(\mathbb{1}-\mathbf{K H})(\mathbb{1}-\mathbf{K H})^{T} \\
& =F(\mathbf{K}) \cdot \Gamma_{n}+G(\mathbf{K})=\Phi\left(\mathbf{K}, \Gamma_{n}\right) .
\end{aligned}
$$


555 Suppose that $\mathbf{K}$ is stabilising, then $\Gamma_{n} \rightarrow \Gamma(\mathbf{K})$ which is a fixed point of (A4), i.e $\Gamma(\mathbf{K})=$ ${ }_{556} F(\mathbf{K}) \cdot \Gamma(\mathbf{K})+G(\mathbf{K})$. Note that $\Gamma(\mathbf{K})$ describes the asymptotic error performance of the 557 feedback $\mathbf{K}$.

${ }_{558}$ We will now show that the output error is able to distinguish (asymptotically) between 559 better and worse feedbacks. For any two symmetric matrices $\mathbf{M}_{1}, \mathbf{M}_{2}$, we write $\mathbf{M}_{1} \geq \mathbf{M}_{2}$ if ${ }_{560} \mathbf{M}_{1}-\mathbf{M}_{2}$ is positive semi-definite but not zero. Let $\mathbf{K}_{1}, \mathbf{K}_{2}$ be two stabilising feedbacks so that ${ }_{561} \Gamma\left(\mathbf{K}_{1}\right) \geq \Gamma\left(\mathbf{K}_{2}\right)$; that is $\mathbf{K}_{2}$ performs better than $\mathbf{K}_{1}$. Further, assume $\left(\mathbb{1}-\mathbf{H K}_{1}\right) \neq 0$ which 562 implies that $\left(\mathbf{A}-\mathbf{K}_{1} \mathbf{H A}, \mathbf{H}\right)$ is observable. (This condition might seem artificial but we will ${ }_{563}$ see later that it is in fact rather natural). We will now show that $\mathbf{H} \Gamma\left(\mathbf{K}_{1}\right) \mathbf{H}^{T}>\mathbf{H} \Gamma\left(\mathbf{K}_{2}\right) \mathbf{H}^{T}$. 564 Note that because $\Gamma\left(\mathbf{K}_{1}\right) \geq \Gamma\left(\mathbf{K}_{2}\right)$ we have

$$
\mathbf{M}_{n}=F^{n}\left(\mathbf{K}_{1}\right)\left\{\Gamma\left(\mathbf{K}_{1}\right)-\Gamma\left(\mathbf{K}_{2}\right)\right\} \geq 0
$$

565 for any $n$ since $F\left(\mathbf{K}_{1}\right)$ preserves positive and negative semi-definiteness. Further, the sequence ${ }_{566} \mathbf{M}_{n}$ is decreasing. To see this, note that it must be monotone since

$$
\mathbf{M}_{n+1}-\mathbf{M}_{n}=F\left(\mathbf{K}_{1}\right)\left\{\mathbf{M}_{n}-\mathbf{M}_{n-1}\right\}
$$

567 and again $F\left(\mathbf{K}_{1}\right)$ preserves definiteness. It cannot be increasing though since $\mathbf{K}_{1}$ is stabilising 568 and hence $\mathbf{M}_{n} \rightarrow 0$. Therefore $\mathbf{H M}_{n} \mathbf{H}^{T} \geq 0$ and decreasing.

569 Assuming $\mathbf{H} \Gamma\left(\mathbf{K}_{1}\right) \mathbf{H}^{T}=\mathbf{H} \Gamma\left(\mathbf{K}_{2}\right) \mathbf{H}^{T}$ would then imply

$$
\begin{aligned}
0=\mathbf{H M}_{n} \mathbf{H}^{T} & =\mathbf{H} F^{n}\left(\mathbf{K}_{1}\right)\left\{\Gamma\left(\mathbf{K}_{1}\right)-\Gamma\left(\mathbf{K}_{2}\right)\right\} \mathbf{H}^{T} \\
& =\mathbf{H}\left(\mathbf{A}-\mathbf{K}_{1} \mathbf{H A}\right)^{n}\left(\Gamma\left(\mathbf{K}_{1}\right)-\Gamma\left(\mathbf{K}_{2}\right)\right)\left(\mathbf{A}-\mathbf{K}_{1} \mathbf{H A}\right)^{n T} \mathbf{H}^{T}
\end{aligned}
$$

${ }_{570}$ for all $n$. Now using the spectral decomposition of $\mathbf{M}_{0}=\Gamma\left(\mathbf{K}_{1}\right)-\Gamma\left(\mathbf{K}_{2}\right)$,

$$
\mathbf{M}_{0}=\sum_{i=1}^{d} \lambda_{i} v_{i} v_{i}^{T}
$$

${ }_{571}$ where $\lambda_{i}$ are the eigenvalues of $\mathbf{M}_{0}$ and $v_{i}$ are the corresponding eigenvectors, we see that

$$
0=\mathbf{H M H}^{T}=\sum_{i=1}^{d} \lambda_{i}\left(\mathbf{H}\left(\mathbf{A}-\mathbf{K}_{1} \mathbf{H A}\right)^{n} v_{i}\right)^{2}
$$

${ }_{572}$ for all $n$. Since $\mathbf{M}_{0} \neq 0$, there is a $\lambda_{j}>0$ and hence

$$
\mathbf{H}\left(\mathbf{A}-\mathbf{K}_{1} \mathbf{H A}\right)^{n} v_{j}=0 \quad \forall n
$$


573 which contradicts the observability of $\left(\mathbf{H}, \mathbf{A}-\mathbf{K}_{1} \mathbf{H A}\right)$. This shows that $\mathbf{M}_{0}=0$ finishing 574 the proof.

575 From the preceding arguments, it follows that any minimiser of the output error must be 576 the asymptotic Kalman gain. To see this, assume $\mathbf{K}_{2}$ is the Kalman gain while $\mathbf{K}_{1}$ optimises ${ }_{577}$ the output error $\mathbf{H} \Gamma(\mathbf{K}) \mathbf{H}^{T}$. By definition of the kalman gain, $\Gamma\left(\mathbf{K}_{1}\right) \geq \Gamma\left(\mathbf{K}_{2}\right)$, and the ${ }_{578}$ preceding discussion shows that $\Gamma\left(\mathbf{K}_{1}\right)=\Gamma\left(\mathbf{K}_{2}\right)$ if $\left(\mathbb{1}-\mathbf{H K}_{1}\right) \neq 0$.

579 To check that this is true, use that the asymptotic output error satisfies

$$
\mathbf{H} \Gamma(\mathbf{K}) \mathbf{H}^{T}=(\mathbb{1}-\mathbf{H K})^{2}\left\{\mathbf{H} \Gamma(\mathbf{K}) \mathbf{H}^{T}+\rho^{2} \mathbf{H} \mathbf{H}^{T}\right\}+\sigma^{2}(\mathbf{H K})^{2} .
$$

${ }_{580}$ Taking the derivative with respect to $\mathbf{K}$ at $\mathbf{K}_{1}$ and using the optimality yields the condition

$$
\mathbf{H K}_{1}=\frac{\mathbf{H} \Gamma\left(\mathbf{K}_{1}\right) \mathbf{H}^{T}+\mathbf{H H}^{T} \rho^{2}}{\mathbf{H} \Gamma\left(\mathbf{K}_{1}\right) \mathbf{H}^{T}+\mathbf{H H}^{T} \rho^{2}+\sigma^{2}}
$$

${ }_{581}$ so $\mathbb{1}=\mathbf{H K}_{1}>0$. As a final remark, $\mathbb{1}-\mathbf{H K}=0$ implies that $y_{n}=\eta_{n}$ (check example (22) ${ }_{582}$ for constant $\mathbf{K}$ ), that is the data assimilation simply reports back the observations.

\section{${ }_{583}$ REFERENCES}

${ }_{584}{ }^{1}$ C. M. Bishop, Neural Networks for Pattern Recognition (Oxford University Press Inc., 585 1995).

${ }_{586}{ }^{2} \mathrm{~B}$. Efron, "How biased is the apparent error rate of a prediction rule?" Journal of the

${ }_{587}$ American Statistical Association 81, 461-470 (1986).

${ }_{588}{ }^{3}$ T. Hastie, R. Tibshirani, and J. Friedman, The Elements of Statistical Learning: Data 589 Mining, Inference and Prediction (Second Edition) (Springer-Verlag, 2009).

${ }_{590}{ }^{4} \mathrm{~B}$. Efron, "The estimation of prediction error: Covariance penalties and cross-validation," 591 Journal of the American Statistical Association 99 (2004).

${ }_{592}^{5}$ E. Kalnay, Atmospheric Modeling, Data Assimilation and Predictability, 1st ed. (Cambridge ${ }_{593}$ University Press, 2001).

${ }_{594}{ }^{6}$ G. Wahba, D. R. Johnson, F. Gao, and J. Gong, "Adaptive tuning of numerical weather prediction models: Randomized GCV in three- and Four-Dimensional data assimilation," Monthly Weather Review (1995).

${ }^{7}$ G. P. Cressman, "An operational objective analysis system," Monthly Weather Review 87, 367-374 (1959). 
604 Review 98, 875-883 (1970).

${ }_{605}{ }^{11}$ A. Lorenc, "A global three-dimensional multivariate statistical interpolation scheme," 606 Monthly Weather Review 109, 701-721 (1981).

${ }_{607}{ }^{12}$ A. H. Jazwinski, Stochastic Processes and Filtering Theory Volume 64 (Academic Press 608 Inc., 1970).

${ }_{609}{ }^{13}$ A. Pikovsky, M. Rosenblum, and J. Kurths, Synchronization: A Univeral Concept in ${ }_{610}$ Nonlinear Sciences (Cambridge University Press, 2001).

${ }^{611}{ }^{14}$ H. J. C. Huijberts, H. Nijmeijer, and A. Y. Pogromsky, "Discrete-time observers and 612 synchronization," Controlling chaos and bifurcations in engineering systems , 439-455 613 (1999).

${ }_{614}{ }^{15} \mathrm{~S}$. Boccaletti, J. Kurths, G. Osipov, D. Valladares, and C. Zhou, "The synchronization of 615 chaotic systems," Physics Reports 366, 1-101 (2002).

${ }_{616}^{16} \mathrm{~J}$. Bröcker and I. G. Szendro, "Sensitivity and Out-Of-Sample Error in Continuous Time ${ }_{617}$ Data Assimilation," Quarterly Journal of the Royal Meteorological Society 138, 1785-801 618 (2012).

${ }^{619}{ }^{17}$ I. G. Szendro, M. A. Rodrìguez, and J. M. Lopez, "On the problem of data assimilation 620 by means of synchronization," Journal Of Geophysical Research 114, D20109 (2009).

${ }^{621}{ }^{18}$ S.-C. Yang, D. Baker, and H. Li, "Data Assimilation as Synchronization of Truth and 622 Model: Experiments with the Three-Variable Lorenz System," Journal of the Atmospheric 623 Sciences 63, 2340-2354 (2006).

${ }^{624}{ }^{19}$ R. Dorf and R. Bishop, Modern Control Systems Tenth Edition (Pearson Education Inc., $6252005)$.

${ }_{626}^{20}$ B. Anderson and J. Moore, Optimal Filtering (Dover Publications Inc, 1979).

${ }^{627}{ }^{21} \mathrm{~W}$. F. Arnold III and A. J. Laub, "Generalized eigenproblem algorithms and software for ${ }_{628}$ algebraic riccati equations," Proceedings of the IEEE 72, 1746-1754 (1984). 